\title{
T1-Hyperintense Plaques on Intracranial-versus-Extracranial Vessel Wall MRI
}

W e read with great interest the recent article by Zhu et $\mathrm{al}^{1}$ regarding the association between T1-hyperintensity of basilar artery stenosis as detected by intracranial vessel wall MR imaging, presumably intraplaque hemorrhage (IPH), and cerebral infarction in the basilar artery territory. T1-hyperintense plaques as a marker of IPH have been validated in the extracranial carotid artery using carotid endarterectomy specimens and have been studied extensively in various populations. In contrast, data on similar lesions in intracranial arteries are rather limited. The study by Zhu et al highlighted the clinical relevance of T1-hyperintensity in intracranial atherosclerotic disease, which should call for further investigations of its etiology and pathophysiology, especially considering the similarities and differences compared with T1-hyperintensity in carotid artery disease.

In the study by Zhu et $\mathrm{al}^{1}$ and virtually all previous studies on intracranial IPH, ${ }^{2,3}$ imaging criteria used for detecting IPH were adopted from carotid MR imaging studies. Radiologic-pathologic correlation studies validating the empiric signal intensity threshold adopted from carotid MR imaging for detecting intracranial IPH are currently lacking. This issue is understandable because it is usually difficult to assess intracranial artery specimens soon after in vivo imaging. However, caution must be exercised because images are generated by different T1-weighted sequences that may produce different T1 contrasts. Although not discussed by Zhu et al, the use of different sequences with different $\mathrm{T} 1$ contrasts may be another potential explanation for the vast differences in the prevalence of IPH in previous studies. ${ }^{1,3}$ In some previous studies, it is unclear whether fat suppression was implemented. In theory, plaques with a high content of lipids may also appear hyperintense on T1-weighted MR imaging. Studies developing and validating imaging criteria for detecting intracranial IPH are warranted.

Notably, the prevalence of IPH in the basilar artery was not different between the low-grade and high-grade stenosis groups, ${ }^{1}$ whereas the prevalence of IPH in the carotid artery often increased with luminal stenosis. ${ }^{4}$ One may explain this finding by a selection bias because the low-grade stenosis group may have included more symptomatic patients. However, the degree of ste- nosis was not associated with symptom status in the study. ${ }^{1} \mathrm{Al}-$ ternatively, this difference between the carotid and basilar arteries may indicate different remodeling mechanisms that exist between different arterial beds. Whether wall area measurements were different between basilar plaques with and without IPH is unknown but a question worth asking.

We congratulate Zhu et $\mathrm{al}^{1}$ for reporting these important data and findings on a relatively unexplored topic. Notably, the association of T1-hyperintensity with cerebral infarction appeared to be even stronger than that of postcontrast enhancement, though most previous studies on intracranial vessel wall MR imaging have only focused on the latter. Certainly, more questions can be raised than answered from the study by Zhu et al, and future efforts are needed to further understand the etiology and clinical significance of T1-hyperintensity on intracranial vessel wall imaging.

Disclosures: Jie Sun-UNRELATED: Grants/Grants Pending: American Heart Association, Comments: I am the principle investigator and have received a grant from the American Heart Association to conduct imaging research on carotid plaque. ** Money paid to the institution of Washington.

\section{REFERENCES}

1. Zhu C, Tian X, Degnan AJ, et al. Clinical significance of intraplaque hemorrhage in low- and high-grade basilar artery stenosis on highresolution MRI. AJNR Am J Neuroradiol 2018;39:1286-92 CrossRef Medline

2. $\mathrm{Xu} \mathrm{WH,} \mathrm{Li} \mathrm{ML,} \mathrm{Gao} \mathrm{S,} \mathrm{et} \mathrm{al.} \mathrm{Middle} \mathrm{cerebral} \mathrm{artery} \mathrm{intraplaque}$ hemorrhage: prevalence and clinical relevance. Ann Neurol 2012;71: 195-98 CrossRef Medline

3. Yu JH, Kwak HS, Chung GH, et al. Association of intraplaque hemorrhage and acute infarction in patients with basilar artery plaque. Stroke 2015;46:2768-72 CrossRef Medline

4. Zhao X, Underhill HR, Zhao Q, et al. Discriminating carotid atherosclerotic lesion severity by luminal stenosis and plaque burden: a comparison utilizing high-resolution magnetic resonance imaging at 3.0 Tesla. Stroke 2011;42:347-53 CrossRef Medline

(D)W. Yuan Department of Radiology Navy Qingdao No. 1 Sanatorium of People's Liberation Army Qingdao, Shandong, China 